\title{
Mortality is not increased with Diabetes in hospitalised very old adults: a multi-site review
}

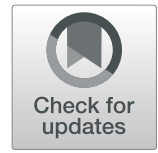

Peter Smerdely ${ }^{1,2}$

\begin{abstract}
Background: Few data exist regarding hospital outcomes in people with diabetes aged beyond 75 years. This study aimed to explore the association of diabetes with hospital outcome in the very old patient.

Methods: A retrospective review was conducted of all presentations of patients aged 65 years or more admitted to three Sydney teaching hospitals over 6 years (2012-2018), exploring primarily the outcomes of in-hospital mortality, and secondarily the outcomes of length of stay, the development of hospital-acquired adverse events and unplanned re-admission to hospital within 28 days of discharge. Demographic and outcome data, the presence of diabetes and comorbidities were determined from ICD10 coding within the hospital's electronic medical record. Logistic and negative binomial regression models were used to assess the association of diabetes with outcome.

Results: A total of 139,130 separations (mean age 80 years, range 65 to 107 years; 51\% female) were included, with $49 \%$ having documented comorbidities and $26.1 \%$ a diagnosis of diabetes.

When compared to people without diabetes, diabetes was not associated with increased odds of mortality (OR: 0.89 SE (0.02), $p<0.001$ ). Further, because of a significant interaction with age, diabetes was associated with decreased odds of mortality beyond 80 years of age. While people with diabetes overall had longer lengths of stay (10.2 days SD (13.4) v 9.4 days SD (12.3), $p<0.001)$, increasing age was associated with shorter lengths of stay in people aged more than 90 years. Diabetes was associated with increased odds of hospital-acquired adverse events (OR: 1.09 SE (0.02), $p<0.001$ ) and but not 28-day readmission (OR: 0.88 SE (0.18), $p=0.523$ ).

Conclusion: Diabetes has not been shown to have a negative impact on mortality or length of stay in hospitalised very old adults from data derived from hospital administrative records. This may allow a more measured application of diabetic guidelines in the very old hospitalised patient.
\end{abstract}

Keywords: Health outcomes, Older adults, Hospital, Diabetes

\section{Background}

Diabetes mellitus is common in developed western nations, with 30 million people diagnosed with diabetes (prevalence 9.4\%) in the US in 2015 [1], and prevalence of 6 and 5.4\% in the UK and Australia, respectively [2].

Diabetes is associated with an increased risk of morbidity and mortality. Diabetes UK reports increased cardiovascular, renal, ophthalmic, peripheral vascular,

Correspondence: Peter.Smerdely@health.nsw.gov.au

${ }^{1}$ Department of Aged Care, St George Hospital, 3 Chapel Street, Kogarah, Sydney, NSW 2217, Australia

${ }^{2}$ School of Population Health, University of NSW, Sydney, Australia neurological and psychiatric disease in poorly controlled diabetes with increased risk of mortality and reduced life expectancy [3]. In Australia, the Australian Institute of Health and Welfare (AIHW) reports death rates being between 1.6 and 2 times higher for those with diabetes than the general population [4].

Hospitalisation in people with diabetes is more likely, more frequent, and with a longer length of stay [5-7]. Consistent with this, in Australia, the prevalence of hospitalisation for any reason of people with diabetes ranges from between 8.9 to $35.1 \%[8,9]$. 
Ageing is strongly associated with the development of diabetes [10]. Likely, the interplay between genetics, environmental factors, and normal ageing is the cause [11]. The prevalence of diabetes increases with age, with 3-5 times the prevalence of diabetes in people over aged 65 years [1,2].

However, detailed data about hospitalisation or outcomes in people with diabetes aged beyond 75 years are presently lacking, with results often grouped as $65+$ or $75+[12]$. Anecdotally, the impression of experienced geriatricians is that diabetes in itself does not confer increased morbidity or mortality in very old adults. This impression occasionally causes conflict with other clinicians or family members when strict diabetic control is relaxed.

This study was undertaken to explore the effect of diabetes diagnosis and age on hospital mortality of patients aged over 65 years using hospital administrative data. The age of 65 years is commonly used to define the older adult. In addition, the hospital outcomes of length of stay (LOS), the development of hospital-acquired adverse events, and unplanned re-admission to hospital within 28 days of discharge was examined.

\section{Methods}

\section{Study design}

A retrospective study was conducted of patients aged 65 years and older admitted for $24 \mathrm{~h}$ or more for acute care in the South East Sydney Local Health District (SESL HD) over 76 months, from 1 July 2012 to 30 September 2018. The study excluded patients presenting for dayonly intervention, outpatient reviews, routine renal dialysis, ambulatory care, and psychiatric management. Subjects were flagged if they had an admission in the 7 years prior to the study period. It was anticipated that patients might have multiple admissions over the study period.

\section{Setting}

SESLHD is located in south east Sydney, Australia, comprising three acute care hospitals: Prince of Wales, St George, and Sutherland. These hospitals provide approximately 1420 beds for south east Sydney, with approximately 139,321 people over 65 years living in the district in 2016. The prevalence of diabetes in the district is $6.4 \%$ and is below the state (NSW) average prevalence of $8.7 \%$ [12].

\section{Data}

Data for this study were based on hospital administrative data, derived from information obtained from the medical record of the patients. These administrative data contain demographic details such as age, sex and race, as well as diagnosis codes, derived from clinician entries in the medical record. This information is held within the "Patient Information Manager" (iPM). iPM is a patient administration system handling all the demographic data, discharge diagnostic codes (DRG), and separation data for all admissions. Study eligibility was based on coding within iPM. Cases were defined as those with a principal or additional diagnosis of diabetes (ICD-10-AM code: E10 Type 1 diabetes mellitus, E11 Type 2 diabetes mellitus, E12 Malnutrition-related diabetes mellitus, E13 Other specified diabetes mellitus, E14 Unspecified diabetes mellitus) as defined by International Classification of Disease 10th Revision Australian Modification (ICD-10-AM). Cases included a new diagnosis during the admission. For comparison, data was also collected from a non-diabetic cohort for the same period, for comparison. A modified Charlson Comorbidity Index (CCI) was generated from ICD-10-AM codes [13, 14]. The CCI was modified by removing the weights associated with diabetes.

The datasets used and/or analysed during the current study are available from the corresponding author on reasonable request.

\section{Hospital-acquired diagnosis (HADx)}

The Classification of Hospital Acquired Diagnoses is a validated system that permits the identification of adverse events that have occurred in hospital during an admission using hospital administrative data $[15,16]$. A hospital acquired diagnosis (HADx) index was generated using a custom written Stata program adapted by the author from existing programs [14]. HADx has been included as a possible confounder and outcome in this study.

\section{Unplanned re-admission}

Unplanned re-admission was defined as being admitted within 28 days following a previous separation. Prearranged or booked admissions within this time frame were excluded.

\section{Statistical analysis}

Data were analysed using Stata Version 16 (StataCorp, College Station, TX, USA). Variables are described using mean and standard deviation, proportion and range. Each separation was analysed as a unique observation. Age, presence of diabetes and the interaction of these two were the variables of interest. The outcome variables of interest were, primarily, mortality during hospital admission (now referred to as mortality), and secondarily, LOS, hospital-acquired disease and re-admission rates. T-tests, Chi-Square tests and Mann-Whitney tests were used where appropriate. Logistic and negative binomial regression models were used to assess association for the binary (mortality, 28-day re-admission and HADX) and count (LOS) outcomes. All models were adjusted for the potential confounding effects of sex, CCI, HADX, and number of admissions before the study period. Effect modification (interaction) was assessed between diabetes and the other variables in the model. A 5\% two-sided significance level was used for main effects and $1 \%$ for interactions. 


\section{Results}

\section{Cohort summary}

There were 191,201 hospital separations recorded between October 2012 and October 2018 in the district. Of these, 145, 090 had a LOS greater than $24 \mathrm{~h}$, from which 139,130 separations were further examined following exclusion of separations for routine renal dialysis and psychiatric management.

Table 1 summarises characteristics of this cohort. A total of $26.1 \%$ of the cohort was recorded as having diabetes. Half of the cohort $(69,513)$ had at least one separation recorded in the 7 years before the study period. People with diabetes had a higher mean number of separations (4.2 (SD 3.9) v 3.5 (SD 3.6), $p<0.001)$. Ages ranged from 65 to 107 years.

There were 848 separate ICD10-AM codes in the cohort, with the five most frequent being: Acute myocardial infarction (code I21); Heart failure (I50); Cerebral infarction (I63); Pneumonia, unspecified organism (J18); and Fracture of femur (S72). These represent $64 \%$ of all diagnoses. CCI scores ranged from 0 to 12 (mean of 1.03 (SD 1.63)), with 55\% scoring 0, 20\% scoring 1 , and a $29 \%$ scoring 2 or more on this measure.

People with diabetes were more likely to have a HADx (complication), see Table 1.

\section{Primary outcome}

The mortality rate for people with diabetes was $8.6 \%$ (v $9.6 \%$ in non-diabetics) (crude odds ratio 0.89, $p<0.001$ ), see Table 2 . When adjusted for age and its interaction with age, the odds ratio was 2.98, and when further confounders were added to the analysis, the odds ratio was 3.15. The analysis demonstrates a significant interaction

Table 1 Cohort Characteristics

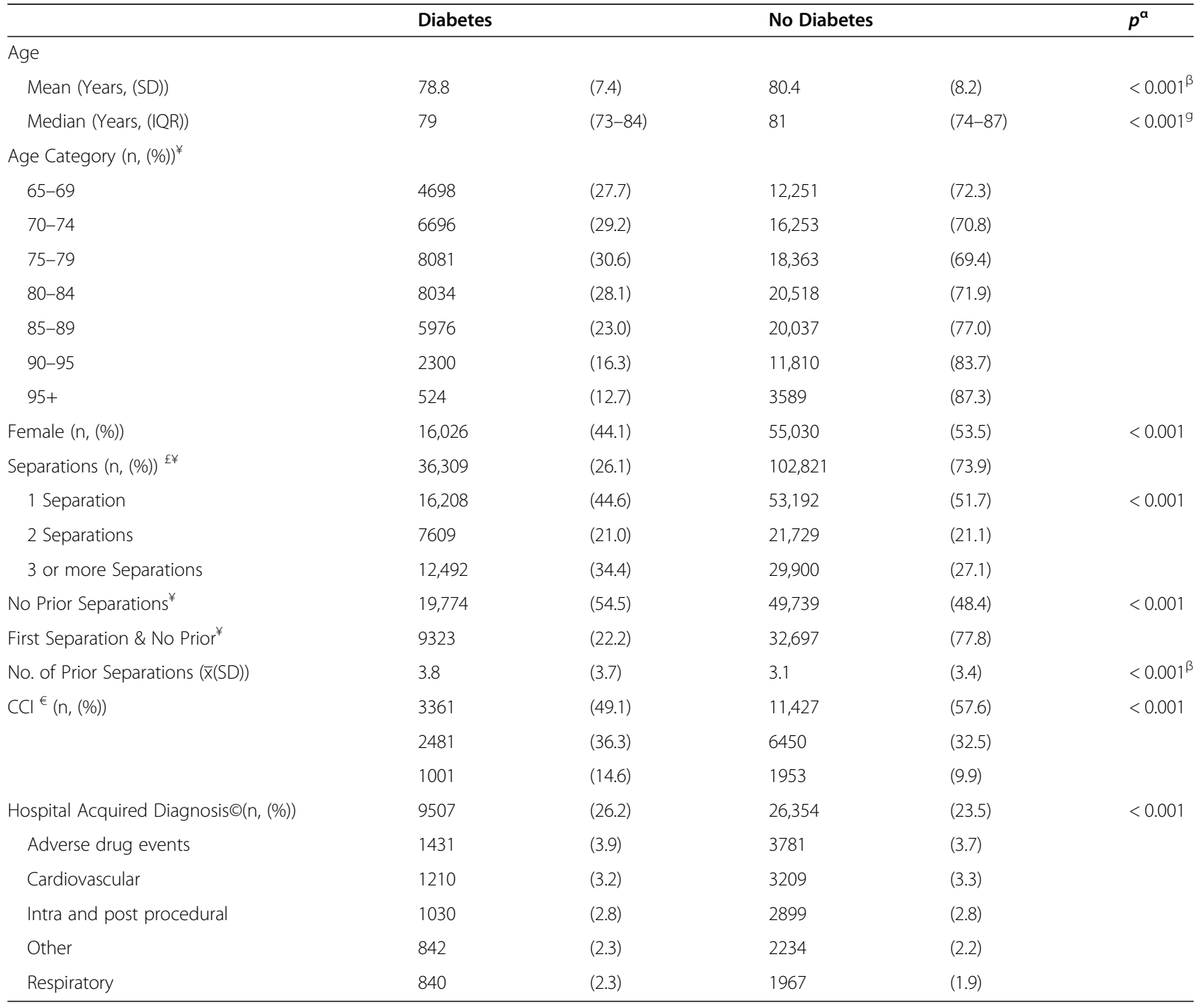

Percentages are column percentages unless indicated. ¥Percentages are row percentages. $£$ Number of separations by each individual in the 6 -year period. $€ C$ harlson Comorbidity Index. aP values are from $X 2, \beta P$ values are from t-test. $\mathbf{g} P$ values are from rank-sum test 
Table 2 Logistic Regression for Mortality

\begin{tabular}{|c|c|c|c|c|c|c|c|c|c|}
\hline & Unac & & & Mod & & & Mod & & \\
\hline & OR & SE & $p$ & OR & SE & $p$ & OR & se & $p$ \\
\hline Diabetes & 0.89 & 0.02 & $<0.001$ & 2.98 & 0.68 & $<0.001$ & 3.15 & 0.72 & $<0.001$ \\
\hline Age & 1.03 & 0.00 & $<0.001$ & 1.03 & 0.00 & $<0.001$ & 1.04 & 0.00 & $<0.001$ \\
\hline Diabetes $x$ age & & & & 0.99 & 0.00 & $<0.001$ & 0.99 & 0.00 & $<0.001$ \\
\hline Female & 1.26 & 0.02 & $<0.001$ & & & & 1.18 & 0.02 & $<0.001$ \\
\hline Prior Separations(n) & 0.94 & 0.00 & $<0.001$ & & & & 0.94 & 0.00 & $<0.001$ \\
\hline Admission Number & 0.92 & 0.00 & $<0.001$ & & & & 0.93 & 0.01 & $<0.001$ \\
\hline $\mathrm{CCl} 1$ & 0.72 & 0.02 & $<0.001$ & & & & 0.71 & 0.02 & $<0.001$ \\
\hline $\mathrm{CCl} 2+$ & 0.86 & 0.02 & $<0.001$ & & & & 0.91 & 0.02 & $<0.001$ \\
\hline HADx & 1.39 & 0.04 & $<0.001$ & & & & 1.45 & 0.04 & $<0.001$ \\
\hline Constant & & & & 0.01 & 0.00 & $<0.001$ & 0.01 & 0.00 & $<0.001$ \\
\hline
\end{tabular}

Abbreviations: OR Odds ratio; se Standard error; CCI Charlson Comorbidity Index; HADx Hospital acquired diagnosis. Models comprise components shown. Unadjusted model used simple logistic regression with death and a single component analysed. Models 1 and 2 used death and components in multiple logistic regression. The analyses include all subjects with admissions before the study period and those with multiple admissions

between age and diabetes. When using the predictive margins generated by the model, mortality in people with diabetes is lower after age 80 years, see Fig. 1a.

Further analysis is shown in Table 3. It adjusts for prior admission, multiple admissions and age. It demonstrates a consistent interaction of diabetes and age across the adjustments where age is not restricted. However, in Model 6 (see Table 3), where age is limited to 80 years or more, diabetes is no longer a significant component of the regression.

\section{Secondary outcomes}

People with diabetes had a significantly longer LOS (10.2 days SD (13.4) v 9.4 days SD (12.3), $p<0.001$ Mann-Whitney). As with mortality, there is an interaction with age with the predicted LOS being lower in people with diabetes beyond the age of 95 years, see Fig. $1 \mathrm{~b}$.

There was a small increase in the odds of developing a hospital-acquired complication in people with diabetes (OR: 1.09 SE (0.02), $p<0.001)$. There was no interaction with age or other confounders. The increased odds persisted throughout the age spectrum.

People with diabetes had a $14.0 \% 28$-day readmission rate compared to $12.1 \%$ in non-diabetics $(p<0.001)$. Diabetes did not affect 28-day re-admission rate in logistic regression when adjusted for age, sex, prior admissions, other comorbidities and HADx (OR: $0.88 \mathrm{SE}$ (0.18), $p=0.523)$.

\section{Discussion}

A commonly held view amongst experienced geriatricians is that strict adherence to onerous diabetic diets with maintenance of tight blood sugar ranges does not confer morbidity or mortality benefits to very old adults, and comes at a cost to patient quality of life. Clinical decisions to relax glycaemic control in the context of significant comorbidity occasionally leads to conflict with other clinicians or patients and their families. To date, there had been no large studies to help inform such management decisions in very old adults. The present study did not find that diabetes was associated with increased odds of mortality in people aged over 65 years with an unadjusted odds ratio of $0.89(p<0.001)$. Diabetes diagnosis has an interaction with age. Accumulating age reduces its impact to the point that a diagnosis of diabetes may predict a better outcome in the very old adults (aged more than 80 years), see Fig. 1a. This may be due to immortal time bias (only the healthiest diabetics survive to ages beyond 80 years) [17, 18]. At the very least, it confirms that diabetes in very old adults does not convey a worse prognosis.

The transition point to the very old adult is arbitrary. By using the median age of the cohort ( 80 years) as this study's transition point, additional analysis, restricted to the oldest, showed no effect of diabetes diagnosis on mortality (see Table 3). Most studies that deal with mortality and diabetes are longitudinal studies [19-23]. There are only a few studies that explore in-hospital mortality associated with diabetes, and these are diseasespecific [24-26]. Two of the studies showed no effect on inpatient mortality $[24,26]$. The third showed an odds ratio of 1.31 (1.04-1.65) for mortality in people with diabetes with foot disease [25]. There may be diseasespecific subsets that are at higher risk, but this was not explored in the present study.

Similar to mortality, diabetes diagnosis had less impact on the length of stay (LOS). Greater LOS may be used as an indicator of greater physical and/or psychological morbidity. Very old adults with diabetes had shorter lengths of stay (see Fig. 1b) despite people with diabetes having overall longer LOS. The impact of diabetes on 

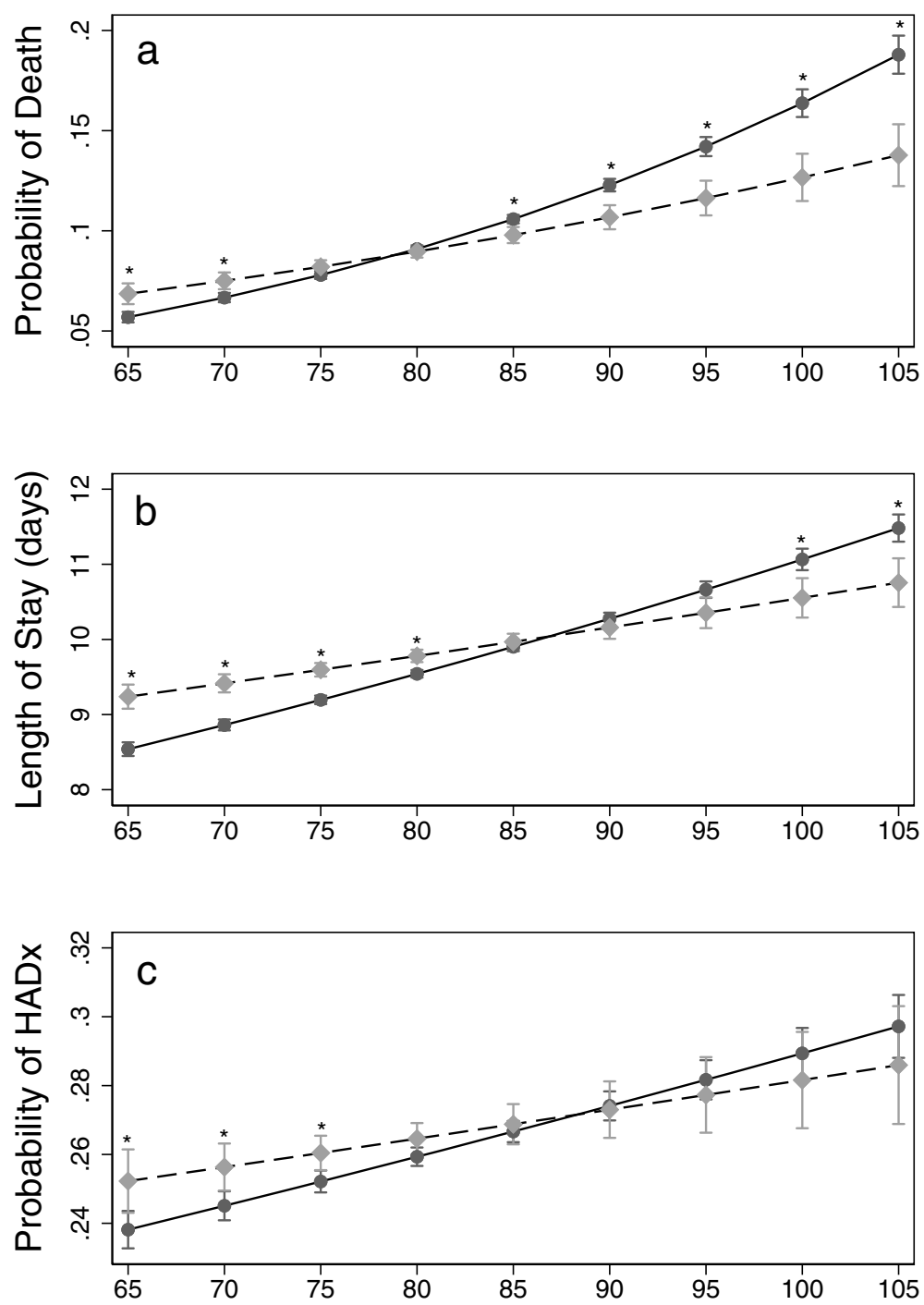

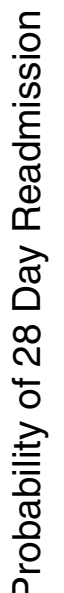

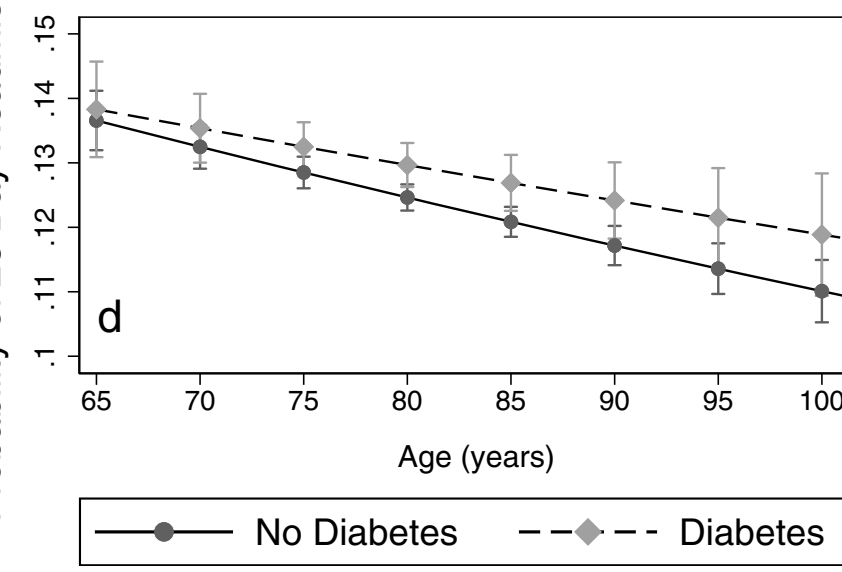

Fig. 1 Predictive Margins of Diabetes by Age against various outcomes. a: Predictive Margins of Diabetes by Age for Death. b: Predictive Margins of Diabetes by Age for Length of Stay. c: Predictive Margins of Diabetes by Age for Hospital Acquired Diagnosis. d: Predictive Margins of Diabetes by Age for 28-day Readmission. Asterisk indicates $p<0.001$ at the age 
Table 3 Logistic Regression for Mortality

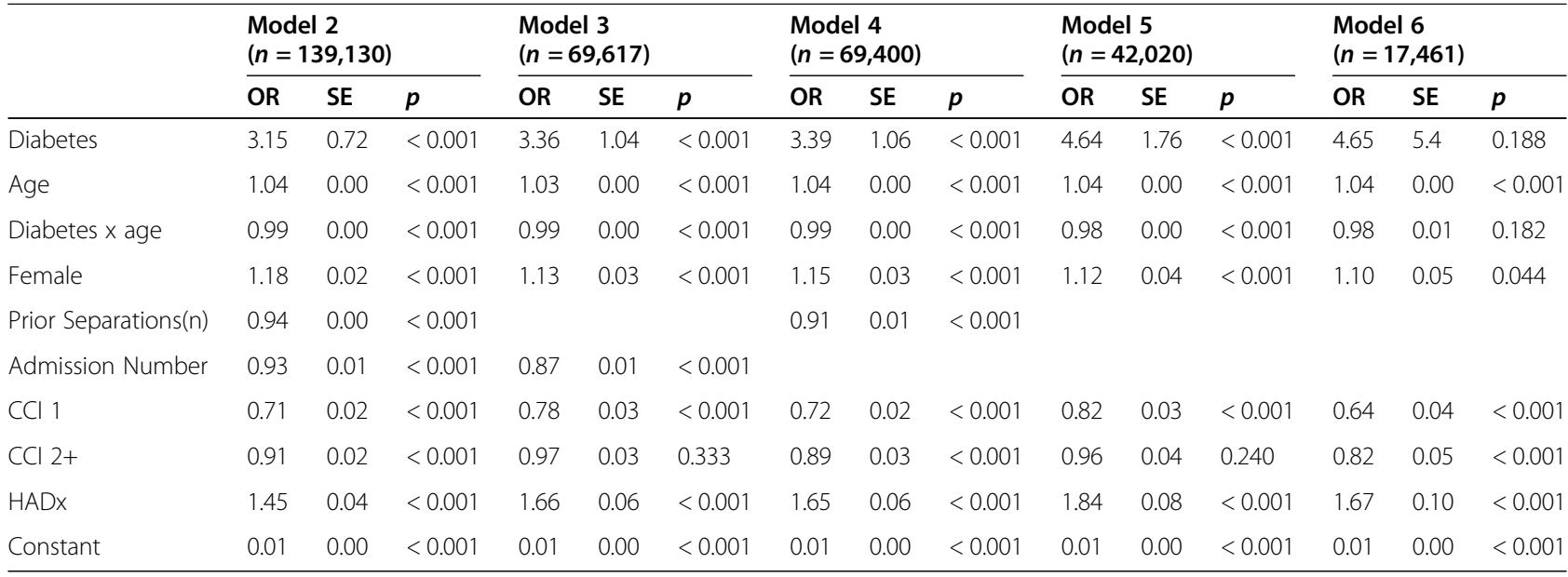

Abbreviations: OR Odds ratio; se Standard error; CCI Charlson Comorbidity Index; HADx Hospital-acquired diagnosis. Models comprise components shown. Model 2 contains all variables without restriction and is the same as Model 2 in Table 2; Model 3 is restricted to those with no prior admission; Model 4 is restricted to the first admission only; Model 5 is limited to those with no prior admission \& first admission only; Model 6 is Model 5 limited to those people older than 80 years (the median age of the cohort)

LOS is consistent with other studies [24, 26-28]. The present study differs from these works by including only people over 65 years and adjusting for the confounding of disease burden by using the CCI. Furthermore, also consistent with these studies is the magnitude of the effect on LOS, which is small.

Hospital-acquired adverse events were higher in the diabetic cohort. It was not modified when adjusting for age. This study used a validated but not extensively used method of detection of hospital-acquired diagnosis [16, 29-33]. The present study also found a much higher proportion of HADX overall, probably related to the examination of an older cohort. By contrast, Cromarty et al. were able to show that $29.3 \%$ of people with diabetes developed hospital-acquired events compared to $13 \%$ of non-diabetics [29]. .

This study demonstrated that the likelihood of readmission within 28 days of discharge was associated with the diagnosis of diabetes, and these odds were not influenced with increasing age. Caughey et al. identified older people (aged over 85 years) with comorbidities as those most likely to be re-admitted within 30 days [34]. The present study adjusted for the presence of comorbidity and found little difference, providing some support for their result. Dungan identified those with poor glycaemic control as those most likely to be re-admitted [35]. Clinical measures were not undertaken in the present work.

The present study does not show why the diagnosis of diabetes does not appear to have an impact on mortality in very old adults. However, it does support clinical decisions to relax glycaemic control goals in this age group, as has been more broadly recommended in international guidelines [36]. There is evidence that rigid control may not have the benefits seen in younger people [37]. A converse viewpoint is that diabetes may not be as harmful in older people, and so its control does not need to be as tight. These considerations will enhance the adoption of a more measured approach to diabetic care in very old adults, particularly those with comorbidities, with less strict blood sugar ranges and a more liberal diabetic diet. These measures may in turn facilitate maintenance of independence and improved quality of life in this age group.

The current study has limitations. It is a retrospective audit of hospital administrative data. Hospital databases have not been designed for clinical investigations. However, the large amounts of data might be used for association. Several validation studies of hospital discharge data exploring diabetes diagnosis (as used in the present study) have been conducted with a diabetes diagnosis giving positive predictive values from 59 to $93 \%$ and negative predictive values from 91 to $99 \%$ [38]. These studies suggest that not having a label of diabetes is more accurate. Improved accuracy of the diagnosis of diabetes can occur by linking hospital datasets with other sets such as prescription databases [39]. Linkage studies were beyond the scope of this study but would merit further research. Moreover, a validation sub-study may be useful. Studies also warn about changes in coding rules occurring over time, such as the changes that occurred in the definitions of diabetes in 2011 [40]. This change has resulted in a decreased level of reporting. This study commenced in 2012 for that reason.

This study chose to analyse each admission as a separate event for its primary outcomes. Thus, individuals may be represented multiple times. However, including or excluding those with multiple admissions could be 
debated. This study analysed with and without multiple admissions, and with and without prior admission (see Table 3). There was no material difference across the different models.

This study did not use specific clinical measures such as medication usage, glycaemic control and measures of frailty. Several small works have examined hospital outcome based on these clinical measures [41-44]. Prospective studies that explore the effects of glycaemic control and frailty on hospital outcomes are needed.

\section{Conclusion}

Consensus amongst clinicians regarding whether to recommend adherence to strict diabetic management regimens in very old patients in hospital is presently lacking. This uncertainty is influenced by concerns of potential impacts on quality of life as well as lack of perceived benefit of potentially onerous management plans. The present work demonstrates that diabetes does not have a negative impact on mortality, LOS or hospital acquired adverse events in the hospitalised very old adults based on data derived from hospital administrative records. This supports clinician decisions to consider less stringent diabetes management plans for their very old patients.

\section{Abbreviations \\ ICD-10: International classification of disease 10th revision; ICD-10- AM: International classification of disease 10th revision Australian modification; SE: Standard error; SD: Standard deviation; p: P-value; US: United States; USA: United States of America; UK: United Kingdom; NSW: New South Wales; iPM: Patient information manager; DRG: Diagnosis related code; CCI: Charlson comorbidity index; HADx: Hospital acquired diagnosis; SESLHD: South East Sydney local health district; IQR: Interquartile range; LOS: Length of stay}

\section{Acknowledgements \\ Many thanks to Dr. Tasneem Mayat (Campbelltown Hospital, Sydney, Australia), for introducing me to the problem; Associate Professor Patrick Kelly (School of Public Health, University of Sydney, Sydney, Australia) for his appraisal of earlier versions of the work and statistical insights; Ms. Cara Dickson for her patience and hard work extracting datasets; and Dr. Nicholas Cordato and Ms. Amy Montgomery for proofreading and suggestions.}

\section{Author's contributions}

The author (PS) confirms that he meets criteria for the role of author as suggested by the International Committee of Medical Journal Editors. PS alone was responsible for the design, analysis and preparation of this article. The author (s) read and approved the final manuscript.

\section{Funding}

This study was undertaken without specific funding.

\section{Availability of data and materials}

The datasets used and/or analysed during the current study are available from the corresponding author on reasonable request.

\section{Ethics approval and consent to participate}

The project was approved by the SESLHD Research \& Ethics Committee (HREC No: 18/007) Consent to participate was not required in this study. The work was conducted on de-identified hospital administrative data.
Consent for publication

Not applicable.

\section{Competing interests}

The author declares no conflict of interest.

Received: 5 June 2020 Accepted: 17 November 2020

Published online: 03 December 2020

\section{References}

1. Centers for Disease Control and Prevention. National Diabetes Statistics Report, 2017. Atlanta, GA: National Center for Chronic Disease Prevention \& Health Promotion; 2017.

2. Australian Institute of Health and Welfare, Cardiovascular disease, diabetes and chronic kidney disease-Australian facts. Prevalence and incidence, in Cardiovascular, diabetes and chronic kidney disease series no. 2. Canberra: AlHW; 2014.

3. Diabetes UK. Facts and Stats. London, UK: Diabetes UK; 2016.

4. Australian Institute of Health and Welfare. Deaths among people with diabetes in Australia 2009-2014. Canberra: AlHW; 2017.

5. Donnan PT, Leese GP, Morris AD. Hospitalizations for people with type and type 2 diabetes compared with the nondiabetic population of Tayside, Scotland: a retrospective cohort study of resource use. Diabetes Care. 2000; 23(12):1774-9.

6. Jiang HJ, et al. Multiple hospitalizations for patients with diabetes. Diabetes Care. 2003;26(5):1421-6.

7. Khalid JM, et al. Rates and risk of hospitalisation among patients with type 2 diabetes: retrospective cohort study using the UK general practice research database linked to English hospital episode statistics. Int J Clin Pract. 2014, 68(1):40-8.

8. Australian Institute of Health and Welfare, Cardiovascular disease, diabetes and chronic kidney disease-Australian facts. Morbidity-Hospital care., in Cardiovascular, diabetes and chronic kidney disease series no. 3. Canberra: AlHW; 2014.

9. Bach $L A$, et al. The high burden of inpatient diabetes mellitus: the Melbourne public hospitals Diabetes inpatient audit. Med J Aust. 2014; 201(6):334-8.

10. Mordarska K, Godziejewska-Zawada M. Diabetes in the elderly. Prz Menopauzalny. 2017;16(2):38-43.

11. Meneilly GS. In: Sinclair AJ, et al., editors. Pathophysiology of diabetes in older people., in Diabetes in Old Age. West Sussex, UK: John Wiley \& Sons; 2017. p. 3-13.

12. Centre for Epidemiology and Evidence. HealthStats NSW. 2018 [cited 2018 19/02/2018]; Available from: www.healthstats.nsw.gov.au.

13. Charlson ME, et al. A new method of classifying prognostic comorbidity in longitudinal studies: development and validation. J Chronic Dis. 1987;40(5): $373-83$

14. Stagg, V., CHARLSON: Stata module to calculate Charlson index of comorbidity, in statistical software components S456719. 2006, revised 13 Sep 2017, Boston College Department of Economics.

15. ACSQHC. Classification of Hospital Acquired Diagnoses. 2018 [cited 2018 28/ 4/2018]; Available from: https://www.safetyandquality.gov.au/our-work/ indicators/classification-of-hospital-acquired-diagnoses/.

16. Jackson TJ, et al. A classification of hospital-acquired diagnoses for use with routine hospital data. Med J Aust. 2009;191(10):544-8.

17. Gleiss A, Oberbauer R, Heinze G. An unjustified benefit: immortal time bias in the analysis of time-dependent events. Transpl Int. 2018;31(2):125-30.

18. Suissa S. Immortal time bias in pharmaco-epidemiology. Am J Epidemiol. 2008;167(4):492-9.

19. Huang ES, et al. Rates of complications and mortality in older patients with diabetes mellitus: the diabetes and aging study. JAMA Intern Med. 2014; 174(2):251-8.

20. Roglic G, Unwin N. Mortality attributable to diabetes: estimates for the year 2010. Diabetes Res Clin Pract. 2010;87(1):15-9.

21. Roglic G, et al. The burden of mortality attributable to diabetes: realistic estimates for the year 2000. Diabetes Care. 2005;28(9):2130-5.

22. Rosenthal MJ, et al. Hospitalization and mortality of diabetes in older adults. A 3-year prospective study. Diabetes Care. 1998;21(2):231-5.

23. Russell $L B$, et al. Hospitalizations, nursing home admissions, and deaths attributable to diabetes. Diabetes Care. 2005;28(7):1611-7. 
24. Ko MC, et al. Effect of diabetes on mortality and length of hospital stay in patients with renal or perinephric abscess. Clinics (Sao Paulo). 2013;68(8): 1109-14.

25. Nirantharakumar $\mathrm{K}$, et al. In-hospital mortality and length of stay in patients with diabetes having foot disease. J Diabetes Complicat. 2013;27(5):454-8.

26. Nirantharakumar $\mathrm{K}$, et al. Impact of diabetes on inpatient mortality and length of stay for elderly patients presenting with fracture of the proximal femur. J Diabetes Complicat. 2013;27(3):208-10.

27. Malone M, et al. The effect of diabetes mellitus on costs and length of stay in patients with peripheral arterial disease undergoing vascular surgery. Eur J Vasc Endovasc Surg. 2014;48(4):447-51.

28. Sajjad MA, et al. Comparison of incidence, rate and length of all-cause hospital admissions between adults with normoglycaemia, impaired fasting glucose and diabetes: a retrospective cohort study in Geelong, Australia. BMJ Open. 2018;8(3):e020346.

29. Cromarty J, et al. Effects of hospital-acquired conditions on length of stay for patients with diabetes. Intern Med J. 2014;44(11):1109-16.

30. Michel J, Nghiem HD, Jackson TJ. Using ICD-10-AM codes to characterise hospital-acquired complications. Health Inf Manag. 2009;38(3):18-25.

31. Parikh $\mathrm{S}$, et al. Exploring in-hospital adverse drug events using ICD-10 codes. Aust Health Rev. 2014;38(4):454-60.

32. Shepheard J, et al. Reconciling hospital-acquired complications and CHADx+ in Victorian coded hospital data. Health Inf Manag. 2019;48(2):7686.

33. Trentino KM, et al. Measuring the incidence of hospital-acquired complications and their effect on length of stay using CHADx. Med J Aust. 2013;199(8):543-7.

34. Caughey GE, et al. Understanding 30-day re-admission after hospitalisation of older patients for diabetes: identifying those at greatest risk. Med J Aust. 2017;206(4):170-5.

35. Dungan KM. The effect of diabetes on hospital readmissions. J Diabetes Sci Technol. 2012;6(5):1045-52.

36. International Diabetes Federation. Managing Older People with Type 2 Diabetes. Brussels, Belgium: International Diabetes Federation; 2013.

37. Sinclair AJ, et al. Evidence-based diabetes care for older people with type 2 diabetes: a critical review. Diabet Med. 2018.

38. Khokhar B, et al. Systematic review of validated case definitions for diabetes in ICD-9-coded and ICD-10-coded data in adult populations. BMJ Open. 2016:6(8).

39. Lipscombe $L L$, et al. Identifying diabetes cases from administrative data: a population-based validation study. BMC Health Serv Res. 2018;18(1):316.

40. Assareh $\mathrm{H}$, et al. Effect of change in coding rules on recording diabetes in hospital administrative datasets. Int J Med Inform. 2016;94(Supplement C): 182-90.

41. Hubbard RE, et al. Comparison of the prognostic importance of diagnosed diabetes, co-morbidity and frailty in older people. Diabet Med. 2010;27(5): 603-6.

42. Jang HC. Sarcopenia, frailty, and Diabetes in older adults. Diabetes Metab J. 2016;40(3):182-9

43. Sinclair AJ, Rodriguez-Manas L. Diabetes and frailty: two converging conditions? Can J Diabetes. 2016;40(1):77-83.

44. Underwood P, et al. Preoperative A1C and clinical outcomes in patients with diabetes undergoing major noncardiac procedures. Diabetes Care. 2014;37(3):611-6.

\section{Publisher's Note}

Springer Nature remains neutral with regard to jurisdictional claims in published maps and institutional affiliations.

Ready to submit your research? Choose BMC and benefit from:

- fast, convenient online submission

- thorough peer review by experienced researchers in your field

- rapid publication on acceptance

- support for research data, including large and complex data types

- gold Open Access which fosters wider collaboration and increased citations

- maximum visibility for your research: over $100 \mathrm{M}$ website views per year

At $\mathrm{BMC}$, research is always in progress.

Learn more biomedcentral.com/submissions 